Voix et Images

volxetimages

\title{
Fuir pour survivre : aliénation et identité chez Michèle Mailhot
}

\section{Jean Anderson}

Volume 10, numéro 1, automne 1984

Littérature canadienne-anglaise

URI : https://id.erudit.org/iderudit/200459ar

DOI : https://doi.org/10.7202/200459ar

Aller au sommaire du numéro

Éditeur(s)

Université du Québec à Montréal

ISSN

0318-9201 (imprimé)

1705-933X (numérique)

Découvrir la revue

Citer cet article

Anderson, J. (1984). Fuir pour survivre : aliénation et identité chez Michèle Mailhot. Voix et Images, 10(1), 93-105. https://doi.org/10.7202/200459ar d'utilisation que vous pouvez consulter en ligne.

https://apropos.erudit.org/fr/usagers/politique-dutilisation/ 


\title{
Fuir pour survivre : aliénation et identité chez Michèle Mailhot
}

\author{
par M. Jean Anderson, Université Queen's
}

Entre 1964 et 1975, Michèle Mailhot fait publier cinq romans : Dis-moi que je vis (1964); le Portique, (1967); le Fou de la reine (1969); la Mort de l'araignée (1972); et Veuillez agréer (1975). Depuis ce dernier roman, elle n'en a plus fait paraître. Cé silence s'explique peut-être en partie par la résolution, dans la Mort de l'araignée et Veuillez agréer, de ce qui nous semble la problématique centrale de son ouvre, l'aliénation ou l'inadaptation de la femme dans la culture québécoise moderne.

C'est en esquissant les traits principaux des romans dont nous allons entreprendre l'étude que nous verrons en effet une certaine homogénéité de contenu: dans le premier texte, Dis-moi que je vis, le personnage qui est aussi la narratrice est une femme, Josée, dans la trentaine, mécontente de son mariage et en quête d'une raison d'être dans un monde qu'elle perçoit comme déshumanisé et machinisé. Dans le Portique, Josée a vingt ans, et tâche de s'intégrer, comme novice, à la vie religieuse dans un cloître qui est pour elle un cadre fermé et mutilant. La Mort de l'araignée raconte le délire fantastique et fantasmatique d'une narratrice anonyme, mariée, qui cherche encore à quarante ans. la lucidité qui la libérera au lieu de la blesser; et Veuillez agréer présente une héroüne divorcée, Judith, dans la quarantaine, qui renonce enfin à un travail et à un mode de vie intolérables parce qu'ils sont aliénants à l'extrême. Ces quatre romans ont donc en commun une héroïne ultralucide, introvertie sinon schizoïde, en pleine crise d'inadaptation ${ }^{1}$.

1. Nous avons laissé de côté le cinquième texte, le Fou de la reine, Montréal, Éditions du jour, 1969, 126 p., parce qu'il diffère, de façon évidente, des autres œeuvres: n'y figure pas la même recherche de l'identité, la même préoccupation à l'égard de la folie et de la condition féminine, et le narrateur paraît être un homme. 
Bref, la problématique de base concerne nettement l'insertion de l'individu dans la société. Dans chacun des quatre textes, Mailhot pose la question de l'aliénation en termes sociologiques; il s'agit en effet moins d'une maladie individuelle que d'une société incapable de reconnaître les besoins de ses membres 2 .

Nous devons aussi nous rappeler que l'individu en question est presque toujours féminin, et que l'optique de l'œuvre nous oblige à considérer le problème de l'aliénation dans une perspective non seulement humaniste mais aussi féministe.

Nous essayerons dans ce travail d'examiner les mécanismes de l'aliénation - les éléments catalyseurs ainsi que leurs conséquences - dans les quatre ouvres de Michèle Mailhot décrites plus haut. Si nous avons déjà indiqué, dans le titre de cette étude, que ìa question de l'identité semble liée à celle de l'aliénation, c'est parce qu'une certaine perspective antipsychiatrique nous paraît avoir défini la schizophrénie de la mêne façon que Mailhot.

La schizophrénie est en effet un concept important chez l'écrivaine : cette maladie mentale, qui résiste à la taxinomie, nous intéresse dans la mesure où elle est liée au problème de l'identité. Laing prétend que le manque de sécurité ontologique empêcherait, chez le schizophrène, la formulation et la réalisation de son identité individuelle ${ }^{3}$. Nous reviendrons plus loin sur cette question, car il nous faudra dans un premier temps aborder les points suivants : à quel degré les sens psychanalytique et sociologique de l'aliénation se rejoignent-ils dans l'œuvre de Michèle Mailhot? Quels sont les mécanismes sociaux désignés par l'écrivaine comme déclencheurs de l'aliénation? Comment celle-ci se manifeste-t-elle dans les œuvres que nous avons choisi d'étudier?

C'est cette deuxième question que nous traiterons en premier lieu, tout en soulignant d'abord un certain nombre de difficultés soulevées par ce terme, l'aliénation. En effet, si nous voulons en relever les traces chez Michèle Mailhot, il nous faudrait en établir la définition, tâche rendue difficile par la complexité et la variété des notions concernant l'aliénation.

Comme nous l'avons indiqué plus haut, il en existe deux définitions de base: la plus répandue, c'est l'aliénation mentale, la folie, "trouble de l'esprit qui rend la vie sociale impossible», état caractérisé par des problèmes de communication et d'interaction sociale, un «comportement inadapté $^{4}$ ». Selon cette description, l'aliéné agit en dehors des normes de com-

2. Cette formule n'est évidemment pas sans rappeler le propos d'E. Fromm, que la société (capitaliste) présente comme normes des états pathologiques, auxquels l'individu sain essaie de s'adapter. Voir The Sane Society, London, Routledge and Kegan Paul, 1956, $370 \mathrm{p}$.

3. Le Moi divisé, Paris, Stock, 1970, 235 p. Voir surtout chapitre 3, "L'insécurité ontologiquen. (Le texte anglais date de 1960.)

4. N. Sillamy, Dictionnaire de la psychologie, Paris, Larousse, 1965, pp. 21-22. 
portement de son groupe social, auquel «il devient étranger ${ }^{5}$ ». Ces éléments de définition mettent en relief la notion d'exclusion par rapport à la norme sociale. Szasz et Foucault, ont exposé dans leurs œuvres contestataires la nature coercitive et apparemment arbitraire de cette étiquette infiniment variable qu'est le terme «aliéné» 6 .

Pour ce qui est des sociologues, l'aliénation a un sens qui, de prime abord, semble différer assez nettement de la définition psychologique traditionnelle, par son insistance sur les causes plutôt que sur les symptômes, Ce sens du terme a ses origines surtout chez Marx, pour qui, dans l'Idéologie allemande, l'aliénation ou "l'extériorisation " se définirait comme résultat du manque de pouvoir de l'individu dans la société capitaliste, où la division du travail inaugure une scission entre les activités physiques et mentales?. Il faudrait cependant ajouter que l'aliénation ne se réduit pas ainsi à cet aspeet unique : comme le précise le philosophe marxiste Schaff,

Le problème de l'aliénation concerne les rapports de l'individu avec la société ainsi qu'avec les divers produits de l'homme comme homme social. (...) Partant donc de l'activité sociale des hommes et de leurs rapports sociaux, il nous faut différencier - suivant en cela Marx - le phénomène de l'objectivation, de la réification de l'activité humaine ainsi que le phénomène de l'aliénation de ses produits, donc par conséquent de l'activité humaine ellemême et enfin de l'homme en tant que tel, l'auto-aliénation ${ }^{8}$.

Est-il possible de voir dans ce quatrième élément, l'auto-aliénation, un équivalent approximatif de cette aliénation mentale, caractérisée par le clivage des fonctions de la personnalité, qui empêcherait l'individu d'entretenir des contacts sociaux ${ }^{9}$ ? Tel est bien le propos de l'école freudomarxiste ${ }^{10}$, et tel semble être aussi, jusqu'à un certain point qu'il nous reste à déterminer, le propos de Mailhot.

5. H. Piéron, Vocabulaire de la psychologie, Paris, P.U.F., 1957, p. 12.

6. Voir surtout T. Szasz, The Myth of Mental Illness, N.Y., Dell, 1961, 337 p., The Manufacture of Madness, N.Y., Dell, 1970, 383 p., et M. Foucault, Histoire de la folie da l'age classique, Paris, Gallimard, 1972, 613 p.

7. Si nous nous arrêtons à cette esquisse de définition, c'est parce qu'elle répond bien au concept de l'aliénation que nous trouverons chez Mailhot.

8. A. Schaff, le Marxisme et l'Individu, Paris, Armand Colin, 1968, pp. 119-120.

9. C'est ainsi que Fromm semble le présenter: «The alienated person cannot be healthy, Since he experiences himself as a thing, an investment, to be manipulated by himself and others, he is lacking in a sense of self. " op. cit., p. 209.

10. Il nous faut signaler ici l'Anti-Oedipe; capitalisme et schizophrénie, de Deleuze et Guattari, Paris, Minuit, 1972, 470 p. Sans appartenir à une école, ces auteurs ne récusent ni Freud ni Marx. Notons aussi la ressemblance entre leur «être-machine» et celui de Mailhot. 
Nous ne tenons pas comme close la tentative de définition que nous venons d'esquisser; nous espérons simplement qu'elle constitue une première réflexion qui nous permettra de cerner un peu mieux les phénomènes de l'aliénation dans les romans de Mailhot que nous pouvons maintenant aborder.

Nous chercherons donc à relever dans les textes choisis les manifestations des deux formes de l'aliénation, mentale et sociale. Bien que Mailhot ne semble pas toujours trancher nettement entre ces deux catégories, il faudra reconnaître que c'est surtout de l'aliénation sociale qu' il est question dans son œuvre.

Pour analyser ces formes d'aliénation, nous suivrons l'ordre de la chronologie fictive qui semble relier les quatre romans: Josée a vingt ans dans le Portique, trente ans dans Dis-moi que je vis; la narratrice de la Mort de l'araignée, quarante ans, comme la Judith de Veuillez agréer.

Ainsi que l'indique tout de suite le titre du roman, il s'agit dans le Portique d'une fuite en un lieu zéro, zone neutre entre la vie dans le monde et celle du cloître. C'est dans cet abri qu'aura lieu une tentative de rejet de la sexualité naissante; mais la dissociation qui valoriserait le spirituel aux dépens du physique s'avère impossible pour la jeune Josée :

Je serai chiffre, formule, sentence, le tout abstrait par l'amour de Dieu... Qu'il croisse et que je diminue. Que je ne sois même plus une femme (...) Rien qu'une âme, une formule, un chiffre, une abstraction.

Femme. Je le suis ce matin dans les douleurs de mon ventre qui éclatent ici comme un superflu tragique. Comment oublier son corps quand les entrailles tirent la cervelle au creux des reins et l'éclaboussent de sang?... intouchable cette fosse de malédictions. Oh! que j'ai mal! Mon Dieu, qu'est-ce que Vous faites de moi? Pourquoi suis-je rejetée dans cette chair qui s'est imposée à moi (le Portique, 32-33)

Pour s'assurer le salut, il faudrait réduire le physique, perçu comme objet et comme extériorité; il faudrait «mortifier ce corps exigeant qui n'en finit pas de réclamer. Le tuer. Être une âme, rien qu'une âme. Je serais invisible. J'échapperais à tous les regards " (LP, 3511). Suivre en cela l'exemple de Monseigneur de Laval, qui «punissait dans son corps les vanités de son esprit " (LP, 23). Agir ainsi, ce serait réaliser 'l'aliénation' totale de son corps, mutilation que Josée ne saurait accepter.

11. Nous employons désormais les sigles suivants:/Le Portique, Montréal, Cercle du Livre de France, 1967, 133 p. - LP;/ Dis-moi que je vis, Montréal, Cercle du Livre de France, 1964, 159 p. - DM;/ La Mort de l'araignée, Montréal, Éditions du Jour, 1972, 102 p. LMA;/Veuillez agréer, Montréal, La Presse, 1975, 145 p. - VA. 
"Personne, sur la terre entière, ne mène une existence aussi insensée » (LP, 87) : cette lutte présentée d'abord comme interne et personnelle, prend bientôt des dimensions plus larges. La doctrine thomiste pesant lourd sur les femmes, "des êtres de péché, des êtres accidentels, des êtres inachevés", $(L P, 88)$, c'est dans le refus et la révolte contre la vie religieuse que Josée est obligée de chercher son intégrité :

Je tiens à ma vie, à ma volonté, à ma faiblesse, à ma bêtise. Je veux assumer à plein ce qu'on m'a donné de si repoussant. J'ai envie d'être une femme authentique, $j$ 'ai envie de m'avilir (LP, 88).

Ainsi pouvons-nous conclure que le Portique explore, pour ensuite les rejeter, les mécanismes, aliénants pour la femme surtout, de la religion catholique. C'est une aliénation qui frappe à la fois l'individu (dans tout son quotidien) et la collectivité :

Que toutes ces femmes muettes, alignées comme des bêtes de somme, me paraissent pitoyables! J'ai honte à leur place, pour chacune d'entre elles, de cette docilité effrayante qui les réduit à n'être plus rien que l'illustration dérisoire d'une vertu inhumaine.

Mon Dieu qu'allez-vous faire de toutes ces femmes mutilées? Elles n'ont plus rien, rien, sauf une générosité proche de la folie (LP, 87).

Rester amènerait nécessairement cette auto-aliénation qui ressemble à la folie, rejet de son intégrité, aliénation d'une partie d'elle-même.

Dans le deuxième texte, Dis-moi que je vis, Josée, quoique plus âgée, cherche encore à se défaire de l'aliénation de son corps, se lavant «avec délices » pour combattre des restes d'un «dégoût» d'origine explicitement religieuse :

Ah! l'aimable revanche contre ces débarbouillages hâtifs du couvent où il fallait, en longues chemises, satisfaire rapidement à l'hygiène, dans de l'eau froide en plus pour être bien sûr d'écarter toute trace de plaisir suspect. Et que s'installent le mépris et la honte. C'est de cela qu'il fallait me laver, de ce dégoût vicieux attaché à mon corps $(\mathrm{DM}, 102) \ldots$

Elle cherche à réparer ce clivage ressenti comme menace et source d'angoisse, afin d'être «réunifiée», pour retrouver "la pure merveille d'exister » (DM, 102, 103). Cette réintégration est pourtant bloquée par un dédoublement tenace et inquiétant: 
J'essayais de m'endormir mais j'étais toujours là pour guetter la profondeur de mon sommeil. Dire : "enfin je dors vraiment» supposait une lucidité de contemplation fort éloignée des rêves indolents. Double, encore mon être dédoublé. Hier, l'esprit survolté faisait des voltiges au-dessus du corps méprisé, aujourd'hui le corps triomphant qui regarde la fourmi esprit ramasser son sable (DM, 107-8).

Il existe dans l'oeuvre de Mailhot de très nombreux exemples d'une telle scission entre le corps et l'esprit, où l'auto-aliénation du sujet est évidente. Cet état divisé, où l'esprit et le corps ne se tiennent plus qu'à peine, ressemble à certains égards à un comportement schizoïde, voire schizophrène. Mais pour Mailhot il ne s'agit pas d'une «maladie» de l'individu; il est difficile, en effet, de considérer l'aliénation mentale, la « folie » décrite ici comme autre chose qu'une inadaptation de l'héroöne à une structure sociale (la vie de la religieuse) elle-même inadaptée aux besoins de l'être humain. L'intégrité dépend d'un lien fragile: «Finfin", et "Bébête» continuent d'entretenir des relations haineuses, comme «deux frères siamois accolés par le front et qui ont envie de s'en cracher plein la figure mais qui hésitent, à cause du retour " (DM, 108).

À cette auto-aliénation par scission, enracinée par la religion, il faut ajouter une deuxième série de structures aliénantes. Dans Dis-moi que je vis c'est le mariage comme institution et ses rapports avec la technologie de la société de consommation qui fait l'objet d'une contestation. Face aux pressions «machinisantes» de cette société, l'être humain risque de se dépersonnaliser: Josée est entourée de machines-machines et d'êtres-machines 12 . Pour s'intégrer dans la culture machinique de l'homme, il faudrait que la femme aussi fonctionne en machine :

Recette du bonheur de l'épouse : tôt le matin, mettez toute la crasse de la semaine dans la merveilleuse machine à laver. Pressez le bouton. Tournez à gauche, mettez la vaisselle souillée dans la merveilleuse machine. Bouton. Déposez le linge purifié dans la merveilleuse machine à sécher. Bouton. Pas de repassage : ces tissus sont tous infroissables. Installez-vous dans une chaise et commencez à être heureuse. Où est le bouton? Zut, on a oublié celui-là. À quoi pensent-ils, les hommes géniaux qui font vivre richement leurs femmes parce qu'ils

12. Josée décrit ainsi son mari, Pierre : «Il faut le voir quand il a congé : une vraie broyeuse de travail qui avale du vide. Il s'affaire comme un bulldozer en mal de montagnes à bousculer» (DM, p. 49). 
inventent pour les femmes encore, des machines et des machines, à quoi pensent-ils donc pour avoir oublié ce dernier petit déclic (DM, 47-8)?

Chez Mailhot, l'aliénation de la ménagère, qui rejoint évidemment le concept de la «robotitude » chez Daly, s'exprime par la déshumanisation ${ }^{13}$ : Louise, la femme parfaite, n'est plus qu' "une manière de statue» (DM, 51). Alors, pour Josée, la nécessité de lutter est claire :

On ne se laisse pas ainsi réduire à l'état d'objet insensible quand on sent chaque pore frémir aux pulsations de la vie (DM, 56).

Ce refus de se laisser machiniser, c'est aussi pour la femme refuser de fonctionner comme objet sexuel, ou machine à faire l'amour. Dans son délire, l'héroïne de la Mort de l'araignée révèle son aliénation en se figurant comme une statute, immobile ${ }^{14}$. Pour la remplacer, son mari se fabrique un robot, et elle se voit métamorphosée en machine, femme-aspirateur avec son fils aspirateur portatif (LMA, 57-8). C'est dans ce texte que jouent tour à tour toutes les formes de l'aliénation susceptibles d'attaquer la femme. Dans le cadre de la religion, du mariage et de la vie sociale, elle se sent mise à part et menacée : «Femme, québécoise, catholique... Vous êtes condamnée, me dit-il, trois fois condamnée» (LMA, 45). Elle reconnaît aussi son auto-aliénation, se voit toujours en double, "observante et observée» (LMA, 17); «S.O.S.: trois ennemis en vue: Dieu, les autres et moi» (LMA, 61).

Si ce livre nous présente surtout un délire où le mariage figure comme source première du conflit, Veuillez agréer dépasse les limites du cauchemardesque «film intérieur" pour retrouver le point de vue analytique de Dis-moi que je vis. Dans ces deux romans à perspectives plus larges, c'est la société tout entière qui est responsable de l'aliénation de tous ses membres. L'être humain est soumis à des conditions sociales qui le condamnent à fonctionner comme une machine, branchée sur cette autre, plus grande, qu'est la société de consommation, «mécanisme implacable» $(V A, 14)^{15}$.

13. Pour M. Daly, la technologie masculiniste chosifie la femme, la transforme en robot, en lui enlevant sa propre réalité. Voir Gyn/ecology: the Meta-ethics of Radical Feminism, Boston, Beacon Press, 1978, pp. 52-56.

14. Ce texte, peut-être le plus réussi de tous les romans de Mailhot, diffère des trois autres étudiés ici par sa forme plus floue, où l'analyse lucide cède la place à la présentation d'une série de scènes de cauchemar qui frôlent la folie.

15. Image qui fait penser au frontispice de $l$ 'Anti-Oedipe, tableau qui représente un garçon branché apparemment à une machine qui se trouve derrière lui. Cette image apparaît aussi chez Fromm, qui dénonce la société technologique pour avoir réduit l'être humain à un état d'appendice à la machine. (The Anatomy of Human Destructiveness, N.Y., Holt Rhinehart Winston, 1973, 521 p.). 
La maison d'édition où travaille Judith, l'héroïne de Veuillez agréer, est un «tube», situé dans une ville avaleuse de l'humanité. C'est un endroit où, pour être «normal», il est nécessaire de s'adapter aux structures rigides de la société en béton:

Penser aux arbres dans le métro, au silence sur la rue Dorchester, au désert dans un centre d'achats, ce n'est plus prier mais délirer.

Me voici donc malade, Seigneur... Pour être normale, je devrais continuer de me boucher les yeux, le nez et les oreilles, suivre leur «conditionnement opérant », garder une attitude respectueuse devant la laideur, le bruit et la pollution, suivre les «schémas organisationnels, réduire ma marge d'oscillation » et arriver au point fixe, neutre et salutaire du oui inconditionnel, critère décisif de la santé mentale (VA, 72).

Nous reprenons ici la question de la folie que cet extrait nous oblige à poser : ce qui ressort de ce texte est une conscience de l'aliénation inévitable - et à laquelle le jargon sociologique ajoute une dimension supplémentaire pour les personnes qui voudraient rester dans le cadre de cette société où la. joie et le bonheur sont inadmissibles. Le sourire provoque une réaction hostile,

... fait grincer la routine chromée, calfeutrée, motorisée, chloroformée, (...) fait douter. Les visages se ferment, se cachent, se mettent à dire, à médire, à contredire, à souffrir. (Il) donne envie d'être un monstre aussi. Et cela ne se fait pas, ne peut pas se faire: donc il faut détruire le monstre gênant (VA, 65):

Il faudrait se conformer, même en se déformant, pour être accepté, pour que son comportement soit sain selon les barèmes sociaux. Ce conformisme essentiel fournit aussi la motivation qui pousserait les femmes à adopter une des deux destinées, déjà décrites par Mailhot comme aliénantes, que la société réservait aux femmes:

...à l'époque de mon mariage nous vivions dans le plus scandaleux conformisme et (...) une prise de conscience personnelle ressemblait à de la provocation. Repenser les schémas traditionnels, c'était presque automatiquement s'aliéner. Pour vivre en paix dans la société, une femme devait alors absolument s'intégrer dans l'un des deux seuls moules préparés pour elle : la vocation religieuse ou le mariage (VA, 103). 
D'un côté, des «moules» dans lesquels les femmes se perdent, et où une première aliénation - du travail et de soi - amène à la longue la folie, aliénation mentale. Ne pas accepter de se soumettre à ces pressions, c'est se mettre à part, s'aliéner les autres. Face à ce dilemme, le texte propose bien une solution, peut-être la seule possible, mais cette solution aussi est nommée aliénation par le groupe social:

Ils disent que la société est malade, mais si tu refuses d'en consommer davantage, ils te croient plus malade encore. La sainteté du non est devenue une aberration mentale; fuir pour survivre dénote un comportement de schizophrène (VA, 72).

Le discours psychanalytique présente-t-il la schizophrénie comme une tentative d'évasion? Il faut noter ici que pour l'école anti-psychiatrique la situation sans issue que nous venons de décrire chez Mailhot est un dilemme schizophrénogène; la "double contrainte» de Bateson désigne le milieu conflictuel de cet individu "schizophrène». En fait, c'est la famille qui serait schizophrène, par cette habitude de transmettre, en même temps, deux ou plusieurs messages contradictoires. Devant l'impossibilité d'obéir, le malade, normalement un enfant, s'évade dans des comportements autistiques ${ }^{16}$. Cooper ajoute un élément à ce concept en créant celui de la «triple contrainte»; il existerait toujours, en effet, un troisième message, une troisième interdiction qui empêcherait le malade de partir pour se retirer tout à fait du conflit en refusant l'autorité familiale, elle-même modelée sur celle du système social. Selon Cooper, ce seraient les efforts de la victime pour se constituer une existence intégrale et autonome, petite révolution au sein de la famille, qui lui mériteraient d'être classée comme "bizarre», et enfin comme schizophrène ${ }^{17}$.

Cette troisième interdiction, contre la fuite, est évidemment d'une très grande importance chez Mailhot. Il semble, en effet, que toute son œuvre tourne autour de ce problème, et qu'elle décrive la lutte d'un sujet (schizophrène potentiel) contre l'aliénation mentale et sociale possibles. Briser la triple contrainte pour s'en aller ou rester, en perdant son identité et son intégrité ainsi que son équilibre mental, telle est la problématique de son cuvre.

L'écrivain paraît aussi poser la question de l'adaptation que nous retrouvons chez Fromm, pour qui la schizophrénie semble être le résultat du refus de l'intégration sociale (qui entraînerait nécessairement l'aliénation sociale): en d'autres termes, le schizophrène, incapable de maintenir ses

16. G. Bateson, «Double Bind», dans Steps to an Ecology of Mind, N.Y., Ballantine Books, 1972, pp. $271-278$.

17. D.G. Cooper, The Language of Madness, London, Allen Lane, 1978, 182 p. 
contacts avec le monde extérieur aliénant, s'en retire dans la maladie mentale, tandis que l'aliéné (sens sociologique) l'est précisément parce qu'il consent à rester dans le cadre d'une société malade. Nous reconnaissons chez Fromm cette contradiction condamnée par Mailhot dans la citation précédente: le rejet de la société aliénante n'est pas forcément un comportement sain, surtout s'il s'agit d'une retraite dans un monde intérieur ${ }^{18}$.

Mailhot, cependant, rejette aussi la tentation de rester «enfermée en soi » $(L P, 19)$; il faudrait plutôt se retrouver et se posséder, en se créant une identité et une intégrité essentielles à sa propre santé :

Disposer de soi, avoir l'usage de soi, s'appartenir (VA, 60). La volupté de se reprendre, de se posséder, de disposer de soi. (...) ....c'est ma vie enfin, ma mienne (VA, 64).

Le «non» schizophrénique chez Mailhot n'est pas une expérience passive, mais une recherche active. Il ne s'agit pas d'une retraite mais plutôt d'une sortie explosive d'un espace trop petit, étouffant et dangereux :

Il faut il faut il faut. Je vais devenir folle, je n'en peux plus : je pars (LP, 127).

Pour ce faire, il est nécessaire de trouver un certain courage :

Se dire qu'on n'a pas l'âme à l'aise dans ces courbatures du corps soumis et avoir le courage d'aller se redresser plus loin, debout dans le soleil, les poignets nus (DM, 59).

Pour la narratrice de la Mort de l'araignée, le moment de la sortie arrive enfin, qui lui permet de réaliser une intégration désirée depuis longtemps:

Pour la première fois, la lucidité me berce au lieu de me blesser, je me salue sans avoir envie de me cracher à la figure, je me rencontre face à face, je me recueille. Mon bien et mon mal enfin réunis... je veux risquer d'être moi (LMA, 101-02).

Après avoir démissionné de son travail détesté, l'héroïne de Veuillez agréer s'en va, libérée d'un poids énorme:

Judith sort du Tube à la manière d'une fusée propulsée par des millions de dollars et qui s'envole vers la lune parce que la terre coûte trop cher (VA, 142).

18. Fromm, op. cit., pp. 206-207. 
Quatre romans, quatre départs : la fuite est bien une constante dans l'œuvre de Michèle Mailhot. Même si ces textes s'arrêtent là où commencerait la vie, enfin intégrée, des personnages principaux, comme si franchir les barrières importait plus que la liberté ainsi obtenue. Les quatre romans étudiés sont remarquables par leur rejet de ces espaces fermés que sont le cloître, la maison conjugale et le bureau à Montréal. Il ne faut absolument pas voir dans la fuite des héroïnes de Mailhot la recherche d'un lieu familier, ni la retraite par démission désignée par Erikson comme une spécificité du caractère féminin :

Certaines femmes, qui observent et pensent vivement et profondément, ne semblent pas avoir le courage de leur intelligence naturelle comme si elles avaient peur, à l'idée d'une confrontation décisive, d'être trouvées sans « réelle intelligence "... Ainsi sont-elles toujours facilement tentées de retourner à «leur place» chaque fois qu'elles se sentent hors de leur place ${ }^{19}$.

Cet auteur, spécialiste de la question de l'identité, voudrait prouver la préférence «naturelle» de la femme pour les espaces intérieurs, lieux où elle se consacre, évidemment, à ses rôles d'épouse et de mère. En fait, le féminin ne se définirait même pas en lui-même mais devrait s'adapter à l'identité des autres:

Les jeunes femmes se demandent souvent (sic) si elles peuvent «avoir une identité» avant de connaître celui avec qui elles se marieront et pour qui elles créeront un foyer. (...) quelque chose dans l'identité d'une femme doit se tenir ouvert pour les particularités du futur mari et des enfants qu'elle aura à élever ${ }^{20}$...

L'œuvre de Mailhot - comme celle, d'ailleurs, de bien d'autres écrivaines - dénonce cette ouverture à autrui, mari, fiancé spirituel, ou famille, comme un mécanisme de l'aliénation, puisque la femme est dépourvue d'une identité propre. En d'autres termes, il manque à cette personne ce que Laing appelle la «sécurité ontologique 21 ». Ce manque a comme résultat possible l'introversion et l'hypersensibilité aux menaces que sont, pour la victime, les événements quotidiens même les plus banals :

19. E. Erikson, «Féminité et espace intérieur », dans Adolescence et Crise : la quête de l'identité, Paris, Flammarion, 1972, p. 281. Le texte anglais est de 1968.

20. Ibid., p. 302.

21. Laing, op. cit., chapitre 3. 
Si l'individu ne peut tenir pour acquises la réalité, la vitalité, l'autonomie et l'identité de son être et des autres; il devient obsédé par la nécessité de trouver des moyens d'être réel, de se maintenir en vie (et les autres comme lui), de préserver son identité, de s'empêcher de perdre son monde 22 .

Ayant rapproché ces textes d'Erikson et de Laing, il faudrait conclure que les femmes seraient plus portées que les hommes à cette introversion schizoïdique et cela pour des raisons relevant directement du système patriarcal (ni l'un ni l'autre de ces auteurs n'indique cependant l'existence de ce facteur). Dans l'œuvre de Mailhot, l'obsession des héroïnes pour intégrer leur être ressenti comme double ou inadapté nous semble donc trouver ses origines dans le fait qu'elles existent en tant que femmes, c'est-à-dire comme des personnes non-intégrées de par leur position même dans une société masculine.

Nous nous croyons maintenant en mesure de répondre à une des questions posées au début de ce travail, pour dire à quel point les sens psychologique et sociologique de l'aliénation se rejoignent chez Michèle Mailhot. Selon les textes étudiés, l'aliénation mentale, dite folie, est une condition nécessaire à l'adaptation sociale réussie; pour rester dans une société malade, il faudrait devenir malade.

À cette mutilation spirituelle s'oppose cependant une intégrité possible: c'est en fuyant que l'héroöne trouvera la santé. Mais cette action est condamnée, appelée schizophrénie, par une société soucieuse de maintenir ses normes. Ainsi est-il évident que Mailhot refuse, en l'invertissant, la description traditionnelle de la folie comme crise interne de l'individu, pour rejoindre plutôt les écoles de l'anti-psychiatrie existentielle et du freudomarxisme qui insistent sur l'importance des facteurs sociologiques dans la genèse d'une aliénation à la fois mentale et sociale.

Mailhot quitte cependant ces chemins plus ou moins connus dans la mesure où elle présente la fuite comme seul salut possible: si la solution qu'elle propose, la sortie totale vers un espace tout à fait en dehors du système culturel du mâle, paraît extrême, elle s'explique pourtant et par l'énormité de l'enjeu, et par l'impossibilité d'apporter des modifications à ce même système. La "place" de la femme est en effet, comme celle des autres groupes opprimés, une place sans contact avec le pouvoir : 
Les identités nationales, religieuses et autres, données ou conquises, sont produites ou acquises à l'intérieur d'un système de pensée linéaire qui n'évolue que selon les intérêts du pouvoir mâle, ou qui régesse selon la régression des mâles qui contrôlent le pouvoir. Car seuls les hommes ont un rapport d'identification aux institutions auxquelles ils se soumettent ou qu'ils tentent de changer 23 .

Ce texte de l'écrivaine québécoise Nicole Brossard indique que le problème de l'identité est lié à celui de l'identification ; pour Mailhot, la femme ne pourrait s'intégrer dans une société mâle qu'en sacrifiant on intégrité. Si elle tient à la préserver, elle est obligée de renoncer à l'intégration sociale.

Lorsque Judith décide, dans Veuillez agréer, de partir pour vivre à la campagne ${ }^{24}$, c'est pour des raisons auxquelles la poète américaine Adrienne Rich semble faire écho dans ces vers qui pourraient résumer les œuvres de Mailhot que nous venons d'examiner :

Le monde est aux hommes. Mais fini.

D'eux-mêmes, ils l'ont vendu aux machines 25 .

Face à ce milieu déshumanisé et aliénant, l'héroïne de Michèle Mailhot partira à la recherche non seulement d'une chambre à soi, mais aussi de son propre jardin à cultiver.

23. N. Brossard, «L'identité comme science-fiction de soi», dans Identités collectives et Changements sociaux, Paris, Privat, 1981. Tome I, pp. 391-395.

24. Il est intéressant de noter la fréquence du thème de l'évasion vers la nature chez les écrivaines, dont, au Canada récemment, M. Atwood, Surfacing, Toronto, McLelland and Stewart, 1972, 192 p.; M. Laurence, The Diviners, Toronto, McLelland and Stewart, 1974, 382 p.; M. Engel, Bear, Toronto, McLelland and Stewart, 1976, 141 p.; J. Barfoot, $A b r a$, Toronto and New York, McGraw-Hill Ryerson, 1978, 199 p.; A. van Herk, Judith, Toronto, McLelland and Stewart, 1978, $190 \mathrm{p}$. Chez les anglophones, on retrouve cette obsession de la fuite (au sens positif) au niveau du récit alors que l'éclatement chez les francophones se réalise plutôt au niveau de l'écriture même. Voilà une question qui mériterait une étude à part.

25. Vers tirés de «Waking in the Dark», dans Diving into the Wreck, Poems 1971-1972, New York, Norton, 1973, 62 p. La traduction est de nous. 